\title{
openheart View point on social media use in interventional cardiology
}

\author{
Mirvat Alasnag, ${ }^{\oplus 1}$ Mamas Mamas, ${ }^{2}$ David Fischman, ${ }^{3}$ Salvatore Brugaletta, ${ }^{4}$ \\ Jordan Safirstein, ${ }^{5}$ Pascal Meier, ${ }^{6,7}$ Vijay Kunadian, ${ }^{8}$ Lukasz Koltowski, ${ }^{9}$ \\ Sheila Sahni, ${ }^{10}$ Chadi AIRaies, ${ }^{11}$ Michael Gibson ${ }^{12}$
}

To cite: Alasnag M, Mamas M, Fischman D, et al. View point on social media use in interventional cardiology. Open Heart 2019;6:e001031. doi:10.1136/ openhrt-2019-00103

Accepted 26 April 2019

Check for updates

(C) Author(s) (or their employer(s)) 2019. Re-use permitted under CC BY-NC. No commercial re-use. See rights and permissions. Published by BMJ.

For numbered affiliations see end of article.

Correspondence to Dr Mirvat Alasnag; mirvat@ jeddacath.com

\section{INTRODUCTION}

The dissemination of scientific developments has conventionally been through two main platforms: medical journals and scientific conferences. In 1665, the Royal Society released the first medical journal, Philosophical Transactions. In 1812, the New England Journal of Medicine published its first edition. As a means to widely circulate advances in medical science in the 19th and 20th centuries, multiple medical journals focusing on medical subspecialties were subsequently founded to share newly discovered data.

The European Society of Cardiology was established around World War II. On 29 January 1949, 14 National Societies established the Board and laid down its by-laws. The following year (1950), the First General Assembly, comprised of 200 people, met in Paris for the World Congress of Cardiology and discussed general cardiology topics. The field of interventional cardiology was born following Andreas Gruentzig's first successful coronary angioplasty on 16 September 1977 using a balloon dilatation catheter in which he treated a short lesion in the left anterior descending artery. Gruentzig presented the results of his first four angioplasty cases at the 1977 American Heart Association meeting. Between 1978 and 1980, he organised four courses in Zurich, Switzerland, demonstrating his new technique in humans. In 1983, Professor Jean Marco organised the first course on angioplasty with live cases from Toulouse. Over the next 35 years, this course evolved from a French to a European gathering and finally, now an international conference with more than 12000 attendees. ${ }^{1}$

As interventional cardiology became more widespread, due to refinements in stent platforms, pharmacotherapy and advances in other technologies, particularly in the structural arena, societies have sought options to accommodate such growth. One such option includes smaller chapters conducting their own regional or institutional workshops and meetings. With the advent of the World Wide Web, individual researchers have relied on the PubMed Medline search to navigate categorised published material. By 2000, other online search engines, such as Google Scholar, further facilitated this process. Societies and journals have also collectively sought to exploit the web to expand their outreach and membership globally. They established their own websites that detail the table of contents and published articles, commentaries and interviews. In addition, visually or technically demanding specialties, such as cardiac imaging and intervention, have used online websites to allow for interactive case discussions, host videos of procedural techniques and demonstrate complex cases including three-dimensional images that would not be possible using more traditional publication platforms. These images, videos and publication links are archived in the website allowing for streamlined future searches by individual investigators.

More recently, social media has become yet another avenue for the interactive sharing of data. Individuals, societies and journals have adopted Twitter, Facebook, Instagram, Snapchat, LinkedIn and Periscope to 'publish' and promote new research, techniques, devices as well as post links to upcoming issues and meetings and create quizzes. Unlike journals and conferences, these platforms remain largely unregulated. ${ }^{6}$ There are no membership or submission requirements, fees, or processes that check if users are licensed physicians. This has generated controversy over the credibility and influence of social media in the educational process. ${ }^{2}$ The aim of this document is to provide an overview of the use of the various social media outlets. It provides a roadmap to guide the use of social media by clinicians as both an educational 
tool and a medium to discuss cases and to disseminate research. Finally, an outline on the protection of scientific propriety, patient data and patient-physician rights is highlighted.

\section{TYPES OF SOCIAL MEDIA PLATFORMS}

When referring to social media, it is important to recognise that there are multiple outlets each with its unique features.

Twitter is a microblogging service founded in 2006. Posts were initially restricted to 140 characters that doubled to 280 characters in 2017. Service is available in most languages except Japanese, Korean and Chinese. Most users have posted cases, links to publications, promoted meetings or conducted journal clubs. This has also been a medium for debate and critique of published trials. There are over 335 million active Twitter users.

Facebook is a social networking service launched in 2004. It is available in multiple languages and has more than 2.2 billion monthly active users. Unlike most other platforms, it has no limitation of characters or number of photos or videos posted. It allows for the option of live streaming. Many use this medium exclusively for family and friends. Other individuals and societies have accessed Facebook to create events, share cases, live stream meetings and interviews, as well as network with other professionals in the field.

Instagram is primarily a photograph and video sharing service. It was launched in October 2010 exclusively on iOS. By 2012, the Android and Windows applications were released. It is currently owned by Facebook and has over 800 million users. As of 2015, over 40 billion photos have been uploaded. The service allows a photo to be edited, tagged and captioned. Most interventional cardiologists who have used Instagram do so to share cases and angiograms or announce upcoming workshops and courses.

LinkedIn is a business service operating through a website and mobile application. It was founded in 2002. Most equate this platform with a public curriculum vitae that recruiters, meeting organisers, industry and institutions target to identify persons of interest. It is multilingual and operates across all continents. It has 500 million members in 200 countries. Lately, it too has become a source for sharing cases and meeting details.

Periscope is a live video streaming application and is a subsidiary of Twitter released in 2015. Although most use simple handheld devices, professional vision mixing technology is possible including landscape view. This has been used to live stream to a global audience.

Snapchat is a multimedia messaging application. It was released in 2011. The principal feature is that pictures, videos and messages are only available for a short time. It also allows elaborate creative editing of the captured photographs and videos. It eventually added the concept of stories that chronicles events. It has reached 187 million daily users. This platform is often used by cardiologists for chats with patients or groups. The deleted conversation serves to maintain the supposed confidentiality of the conversation.

\section{CURRENT USES OF SOCIAL MEDIA IN INTERVENTIONAL CARDIOLOGY}

The interventional cardiology arena has exploited social media through several mechanisms. Below are the most common uses (online appendix 3):

1. Demonstration and expansion of techniques through discussions and videos that highlight procedural steps is the cornerstone of social media use by interventional cardiologists. Examples include the \#RadialFirst and \#ldtra (left distal transradial) hashtags which have been successful in promoting safe access site procedures while at the same time referencing data to support a change in practice. Many procedural tips and tricks as well as shared experiences are included. Other examples are the \#PercAx which demonstrates the safe use of the axillary artery as an alternative large bore access while \#CTO101 accentuates new techniques and data with respect to revascularisation of chronic total occlusions. Trainees, in particular, have found social media valuable as it enables them to ask experts in such procedures directly and their educational experience is no longer limited to their home institution. The hierarchy is maintained, but the outreach has expanded to all corners of the globe.

2. Social media has been used for the recruitment of research sites as well as for patient recruitment into randomised controlled trials (eg, HYBRID Trial (Percutaneous coronary intervention in stable angina Trial)). It has also been used as a tracking tool for ongoing trials. One example is the ISCHEMIA trial, International Study of Comparative Health Effectiveness with Medical and Invasive Approaches, which has been the subject of heavy criticism on Twitter. In turn, expert perspectives were published to address the concerns raised and to clarify the ISCHEMIA trial design. ${ }^{3}$

3. Journal Clubs and TwitterChats are becoming more commonplace with robust discussions regarding recent publications focusing on study design, analysis and impact on clinical practice. Recent discussions of trials, such as ORBITA (Percutaneous coronary intervention in stable angina), ISCHEMIA and FAME (Fractional Flow Reserve versus Angiography for Guiding Percutaneous Coronary Intervention), included thousands of posts with a discussion of each trial in far greater detail than would be possible at either conferences or in the scientific literature. All these have associated hashtags that track the discussions. Many journals have adopted this journal club approach to cover key areas in interventional cardiology. As an example, a recent EuroIntervention TwitterChat focused on Bifurcation Strategies. ${ }^{4}$ This chat provided an overview of the different strategies underpinning nuances and 
refinements in the techniques that may not be evident from original research.

4. Complex cases including images and videos fielding expert opinions on treatment options and strategies for structural, peripheral and complex coronary anatomies have been routinely posted. Polls regarding treatment options and links to supporting data have been included in these threads.

5. Tweetorials, which are a streamlined review of basic concepts, cover numerous topics including basics of statistics, shock, multivessel percutaneous coronary interventions and treatment of critical limb ischaemia have become mainstream on social media outlets.

6. Patient-targeted campaigns to increase awareness of health-related issues are often encountered. One example is the May Measurement Month campaign for hypertension. The focus of this campaign is increasing global awareness of hypertension and screening mechanisms, lifestyle changes and treatment options, including interventional technologies, such as renal denervation.

7. Campaigns targeting the medical community have also used social media to increase the awareness and trigger a conversation. These include conversations about diversity and sex discrimination in the medical community. One such campaign is the Ohio State Medical School led by the Dean of Admissions, Dr Quinn Capers, who provided a concrete outline and steps to overcome discrimination in medical schools and fellowship admission processes.

8. More recently, the concept of archiving interesting cases and providing a focused discussion was created on Twitter. It's called the Tweetbook: Cardiovascular Interventions. This was an innovation by Dr David Fischman and managed by the wikidoc.org team.

9. Social media has allowed members of the community to follow the latest trials/news from major conferences without attending in person. Often slides, brief commentary and updates are shared across the globe even if the hall holds less than 100 attendees. In addition, live streaming of important late breaking trials or live cases has allowed for a wider audience to engage from remote sites.

\section{JUSTIFICATION FOR GUIDANCE}

With such a large and expanding number of users and applications, it is important to ask why the medical community needs guidance for social media engagement. As physicians, we are required to maintain professionalism upholding standard of care practices that ensure patient safety and privacy. In addition, promoted practices must be evidence-based. The following observations delineate some of the nuances of public network services:

1. All platforms are public. This means anyone has access to our posts. These include patients and their family members, co-workers, editors, journalists, reporters, lawyers, employers and administrators, members of the medical industry, students, trainees and researchers.

2. In this day and age, we should assume that all posts and tweets are permanent despite our presumed ability to delete or edit them. Sophisticated software allows for screenshots or extraction of the post for future reference.

3. The limitation of characters in tweets can lead to misinterpretation. It isparticularly important to note that engagement is often with individuals of variable levels of training, experience, literacy and fluency. In addition, unlike personal direct conversations, the emotional display is not captured. This doesn't allow users to appropriately convey the tone or message intended with little opportunity for clarification.

4. The current generation is using social media and it is expected that there will be an unprecedented expansion with more platforms and greater functionality that will match the developments in information technology and its infrastructure and software.

\section{PROPOSED STRATEGIES TO ENSURE PROFESSIONALISM}

On the basis of the aforementioned, best practices for social media use by clinicians are outlined below.

1. Institutional by-laws and regulations: It is important that users familiarise themselves with and comply with their own employer's/institution's social media regulations and code of conduct particularly as it applies to privacy rights.

2. Industry: It is important to disclose any conflict of interest at the outset when engaging in discussions addressing specific products. This includes serving on advisory boards, proctorship programs or in any other capacity that may be perceived as a conflict. This is standard to all disclosures currently required by all journals, conferences and guidelines.

3. Journals and societies: When serving as a board member of a journal or society (including a social media editor), users are encouraged to adhere to the mission and vision of the entity they represent. It is important to distinguish their own personal views and opinions from those representative of the journal or society they serve.

4. Academia and research:

a. Similar to presentations in meetings, it is appropriate for users to reference and credit the authors, trials and publications when posting data, graphs, images or excerpts on social media avoiding plagiarism.

b. Users are encouraged to link specific citations or tag relevant experts, authors and editors pertinent to the work being discussed.

c. Professional discourse is expected when debating or critiquing a scientific topic. Similar to any scientific format, a respectful choice of language and style are required. Validation of statements with appropriate references including guidelines is strongly advised. 
d. It is encouraged that educational activities, such as Journal Clubs, Quizzes, Chats and Tweetorials, include experts in the field for maximum educational benefit.

e. Whenever possible, society or journal sponsored educational activities should provide CME credits for participants.

f. Strict adherence to the embargo deadlines set by conference organisers, societies or journals is mandatory. Note that the premature release of results and conclusions is considered a violation of the embargo principle.

5. Case reports:

a. When sharing cases, it is mandatory to maintain patient confidentiality at all times in compliance with general privacy guidelines. All patient identifying information must be removed. Consent from the operator (if not the individual posting on social media) is strongly recommended. Consent from the patient and facility is contingent on the individual institutional regulations.

b. Off-label use of a product should be clearly labelled as such.

c. Providing a concise relevant history, clear images/ videos and explicit conclusion with an educational take-home message is recommended when possible.

d. Clinicians/operators who share their experience and tips/tricks with the wider audience should clearly outline any potential complications. Acknowledging the lack of data or guidelines for novel techniques must be clearly communicated. Attempting to reproduce novel procedures based on social media case reports without appropriate guidance/proctorship should be discouraged as it subjects both the operator and patient to risk.

e. Where possible, a disclaimer should be added when a disturbing image is posted (this is applicable to any sensitive material).

6. General public newspapers and television outlets: Links to articles and interviews in general should use language and terminology understandable and sensitive to the general public. Conduct yourself as you would in a professional society. Trolling, politicizing and polarizing views are not recommended.

7. 6. General use: For individuals, social media can contribute to one's professional career both in a positive and negative manner.

a. It is advisable to maintain a profile that identifies your areas of expertise and interests and portrays your genuine self (don't brand yourself as someone you are not).

b. It is advisable to recognise and exploit your strengths.

c. It is advisable to maintain a professional and respectable demeanour.

d. It is advisable to retract and apologise for any error. e. It is advisable to be clear and concise. f. It is advisable to allow others a rebuttal and disengage and 'walk away' from a heated discussion (don't be baited into an inappropriate conversation.)

g. It is advisable to challenge the comment, not the individual. Don't respond to a troll in an equally scathing manner, that is, do not be provocative or inflammatory just to remain relevant. It is strongly discouraged to use caustic language and make personal insinuations. An article by Gordan Fraser 'The Twitterization of the Academic Mind' highlights offensive tweets that edge on racism or misogyny or personal accusations that depart from respectable scholarly expression. ${ }^{5}$ As such, it is important to behave, as one would in a professional meeting or at one's home institution when discussing a topic that may not agree on with a colleague. Remaining respectful at all times is fundamental to any professional discourse.

\section{FUTURE DIRECTIONS}

The interventional cardiology community has already embraced social media. It has become an integral part of the continued educational process and the dissemination of knowledge for trainees and established operators. For example, fellows-in-training have populated a list of topics that are regularly covered by experts in the form of Tweetorials and quizzes. Industry leaders have explored the role of social media and slowly started to engage end users by advertising products and training workshops. Clinicians have taken upon themselves to critique publications, a privilege previously reserved for peer-reviewed journals (now referred to as review by the crowd). Finally, journals and societies have recognised the influential role of social media and created accounts to reach out to a wider membership. (appendix 1 and 2: List of relevant hashtags and high-volume accounts.)

In the future, as an educational platform, social media needs further development in several key areas, defined below:

1. Archiving, cataloguing and retrieving posts/conversations: A wide range of material has been discussed across the various social media platforms. A conversation, post or thread may provide links to publications, important summaries and educational tips. The archiving systems of many of these outlets are cumbersome and archaic. The difficulty lies in the format of the outlet itself as well as the variable material (images, interviews, polls, links and conversations). Many users have found difficulty in retrieving old posts. Twitter enabled the Bookmarking option to allow a user to return to a post. This, however, does not permit an advanced search. As medical users, it is important that we explore with the technical support of the social media companies a means to catalogue material and facilitate searches by adding filter features and bookmarking for more recent posts. The hashtag is 
one way to search for a specific topic. Other examples are the Tweetbook, Wikidoc.org and ThreadReader Application for Twitter users. This will be valuable for trainees reviewing for exams, lecturers preparing for presentations and operators strategising for a complex procedure. Lately, it has been suggested to reference tweets for future retrieval as follows: Last name, First name (User Name). 'The tweet in its entirety.' Date, Time. Tweet.

2. Mechanism of editing: Outlets, such as Facebook, Instagram and LinkedIn, allow for editing the posts and marks them as such. Twitter has not enabled the editing feature. Snapchat is limited by time after which a post is automatically deleted, thereby making edits difficult.

3. Continuous medical education credits: Societies and journals are now conducting chats and journal clubs. Many users were also able to keep pace remotely with the late breaking trials and presentations at major meetings through live streaming and posts by designated ambassadors. With live streaming, for example, the social media companies are able to track the number of users as well as the time each individual user was following the streaming or engaging in the discussion. Potentially, vendors awarding CME credits for societies or meetings could obtain this information from the companies to permit issuance of credit hours or fractions of an hour (micro CME, or CME on the fly) for individual followers.

4. Moderating the conversation: Attempts to create online moderators to channel the discussion during a chat requires refinement. As the number of those involved increases, it becomes difficult to maintain a fluid conversation that is directed at the designated experts without 'side tracking' amongst the audience. Delays in posts also slow down the question and answer format. Such dialogue is easier on Facebook that has no limitation of characters and an entire conversation is maintained in the same post. Twitter, on the other hand, may easily stray as users can choose to retweet instead of reply thereby fragmenting the conversation. Webinars with live question and answer sessions remain superior from an organisation point of view and the issuance of CME credits.

5. Critical appraisal: Many discussions dissecting concepts and trials take place on a daily basis on social media. Whether this needs to be streamlined and regulated remains questionable. Many believe it does not as it has levelled the ground and allowed end users (clinicians and operators) to bring their points of view to the forefront. Many have equated it with the floor microphone that allows the audience to address the presenter/lecturer/trialist/expert at the end of their presentation.

6. Scheduling activities: Most outlets do not have a function to schedule an event except Facebook, which allows the creation of an event. This is an untapped function that will allow users to receive notification of upcoming activities and automatically import them into the calendar.

\section{CONCLUSION}

Social media has become a reality connecting the patient, clinician, researcher, industry and general media across multiple platforms and that cannot be ignored by the interventional cardiology community. Many believe it is critical that the field establish a code of conduct that governs the use of the different outlets. Such a code aims to disseminate knowledge and enhance one's individual experience while maintaining patient safety and privacy.

\section{Author affiliations}

${ }^{1}$ Cardiac Center, King Fahd Armed Forces Hospital, Jeddah, Saudi Arabia

${ }^{2}$ Manchester University, Manchester, UK

${ }^{3}$ Sidney Kimmel Medical College at Thomas Jefferson University, Philadelphia, Pennsylvania, USA

${ }^{4}$ Cardiovascular Institute Hospital Clinic, Barcelona, Spain

${ }^{5}$ Gagnon Cardiovascular Institute, Morristown Medical Center, Morristown, New Jersey, USA

${ }^{6}$ Kantonsspital Graubunden, Chur, Switzerland

${ }^{7}$ Cardiovascular, Royal Brompton Hospital, London, UK

${ }^{8}$ Freeman Hospital, Newcastle Upon Tyne, UK

${ }^{9}$ Medical University of Warsaw, Warsaw, Poland

${ }^{10}$ Garden State Med Multi-Specialty Grp PA, Raritan Bay Medical Center, Clark, New Jersey, USA

${ }^{11}$ Cardiovascular Division, Wayne State University School of Medicine, Detroit, Michigan, USA

${ }^{12}$ Cardiovascular Division, Beth Israel Deaconess Medical Center, Boston, Massachusetts, USA

Contributors I attest that all the authors have participated in the elaboration of this manuscript.

Funding The authors have not declared a specific grant for this research from any funding agency in the public, commercial or not-for-profit sectors.

Competing interests None declared.

Patient consent for publication Not required.

Provenance and peer review Not commissioned; externally peer reviewed.

Open access This is an open access article distributed in accordance with the Creative Commons Attribution Non Commercial (CC BY-NC 4.0) license, which permits others to distribute, remix, adapt, build upon this work non-commercially, and license their derivative works on different terms, provided the original work is properly cited, appropriate credit is given, any changes made indicated, and the use is non-commercial. See: http://creativecommons.org/licenses/by-nc/4.0/.

\section{REFERENCES}

1. Serruys PW, Marco J. The history of EuroPCR and why a new journal? Eurolntervention 2005;1:4-5.

2. MiltonP. Do you practice Twitter-Based medicine? Medscape, Blogs: Revolution \& Revelation 2018.

3. Yeh RW. Academic cardiology and social media. Circulation: Cardiovascular Quality and Outcomes 2018;11.

4. Milasinovic D, Wijns W, Ntsekhe M, et al. Step-by-step manual for planning and performing bifurcation $\mathrm{PCl}$ : a resource-tailored approach. Eurolntervention 2018;13:e1804-11.

5. FraserG. The Twitterization of the academic mind. The Chronicle Review 2019.

6. Fox CS, Barry K, Colbert J. Importance of social media alongside traditional medical publications. Circulation 2016;133:1978-83. 